\title{
Serum melatonin is inversely associated with matrix metalloproteinase-9 in oral squamous cell carcinoma
}

\author{
ADINA ELENA STANCIU ${ }^{1}$, ADINA ZAMFIR-CHIRU-ANTON ${ }^{2}$, MARCEL MARIAN STANCIU $^{3}$, \\ ANCA PANTEA-STOIAN $^{4}$, CORNELIA NITIPIR ${ }^{5}$ and DAN CRISTIAN GHEORGHE ${ }^{6}$ \\ ${ }^{1}$ Department of Carcinogenesis and Molecular Biology, Institute of Oncology Bucharest, Bucharest 022328; \\ ${ }^{2}$ ENT Department, Grigore Alexandrescu Children's Emergency Hospital and Coltea Clinical Hospital, Bucharest 011743; \\ ${ }^{3}$ Electrical Engineering Faculty, Politehnica University of Bucharest, Bucharest 060042; \\ ${ }^{4}$ Department of Hygiene, Carol Davila University of Medicine and Pharmacy, Bucharest 050474; \\ ${ }^{5}$ Department of Medical Oncology, Elias University Emergency Hospital, Bucharest 011461; ${ }^{6}$ ENT Department, \\ Maria Sklodowska Curie Children's Emergency Hospital, Bucharest 077120, Romania
}

Received July 29, 2019; Accepted December 12, 2019

DOI: $10.3892 / \mathrm{ol} .2020 .11392$

\begin{abstract}
Matrix-metalloproteinases (MMPs) and their tissue inhibitors (TIMPs) expression levels have been demonstrated to have prognostic value in oral squamous cell carcinoma (OSCC). The present study hypothesized that melatonin, a small lipophilic molecule primarily secreted by the pineal gland, may be able to regulate MMP activity in OSCC progression. This study aimed to investigate the associations between melatonin, MMPs, TIMPs and the histopathological characteristics of patients with OSCC. A total of 40 men with OSCC (mean age, $57 \pm 7$ years) and 30 healthy men (mean age, $56 \pm 5$ years) were enrolled in the present study. Enzyme immunoassays were used to measure the serum levels of melatonin, MMP-9, MMP-2, TIMP-1 and TIMP-2 before and after transoral surgery for OSCC. Serum melatonin level was significantly lower in patients with OSCC compared with controls, both pre-surgery and 2 days after surgery $(\mathrm{P}<0.001)$. In addition, melatonin level was negatively correlated with MMP-9 ( $\mathrm{r}=-0.6371$ ) and the MMP-9/TIMP-1 ratio ( $\mathrm{r}=-0.4700)$, but not with the MMP-2 or MMP-2/TIMP-2 ratio, in patients with OSCC. These results demonstrated that low levels of melatonin and high levels of MMP-9 correlated with large tumors with invasive depth $(r=-0.35$ and $r=0.33)$ and lymph node metastasis $(r=-0.56$ and $r=0.34)$. The results of this retrospective clinical study suggested that melatonin may be considered as a predictive biomarker of tumor growth and metastasis and a potential therapeutic agent for patients with OSCC.
\end{abstract}

Correspondence to: Dr Adina Elena Stanciu, Department of Carcinogenesis and Molecular Biology, Institute of Oncology Bucharest, 252 Fundeni Street, Bucharest 022328, Romania E-mail: adinaelenastanciu@yahoo.com

Key words: oral squamous cell carcinoma, melatonin, matrix metalloproteinase-9, tissue inhibitor of metalloproteinase-1, metastasis, biomarkers

\section{Introduction}

Squamous cell carcinoma of the oral cavity represents the most common type of oral cancer (1). The 5-year survival for oral squamous cell carcinoma (OSCC) was $~ 50 \%$ in 2016, worldwide despite advances in surgical treatment (robotic surgery), postoperative chemoradiotherapy or targeted therapy in locally advanced and recurrent/metastatic disease $(2,3)$. Loco-regional invasion and metastasis to cervical lymph nodes are considered to be the reasons for the poor prognosis of patients with OSCC. It is therefore crucial to understand the underlying mechanisms of metastasis in OSCC and to identify potential novel biomarkers that would allow the earlier detection of cancer and could be used as agents for targeted therapy in OSCC.

Studies from the past 40 years have reported that matrix metalloproteinases (MMPs), a family of zinc-dependent endopeptidases, and the tissue inhibitor of metalloproteinases (TIMPs), are the principal mediators of the alterations observed in the tumor microenvironment during cancer progression, indicating that they are directly involved in the majority of biological processes leading to metastasis (4). MMP expression is controlled by various hormones (estrogen and progesterone), cytokines (interleukins and interferons) and growth factors (epidermal growth factor, fibroblast growth factor, vascular endothelial growth factor and tumor growth factors) $(4,5)$. Loco-regional metastases are hallmarks of OSCC. MMPs degrade the components of the extracellular matrix (ECM) and the basement membranes, allowing tumor cells to migrate and metastasize into other organs via the vascular and lymphatic systems (4). TIMPs are known to inhibit the catalytic activity of MMPs in a 1:1 stoichiometric relationship (6). It has been previously demonstrated that expression levels of MMP-9, also known as gelatinase-B, MMP-2, also known as gelatinase-A, TIMP- 1 and TIMP- 2 are associated with invasion and metastasis in OSCC (6-9).

Melatonin (N-acetyl-5-methoxytryptamine) is an indolic compound primarily secreted by the pineal gland that regulates 
the sleep-wake cycle (10). Small amounts of melatonin are also synthesized in the retina (11), gastrointestinal tract (12), skin (13), bone marrow (14), lymphocytes (14) and gut (12). Melatonin was also recently discovered in serous cells of human salivary glands (15). Previous studies have demonstrated that melatonin is not only a hormone, but is also involved in tumor suppression in various types of cancer, including breast (16), prostate (17), ovarian (18), cervical (18), endometrial (18) and colon (19) cancer, through its anti-proliferative, pro-apoptotic and anti-angiogenic functions. The anti-metastatic role of melatonin in OSCC was mainly investigated using preclinical in vitro and in vivo models (20-23). Numerous studies have reported that melatonin decreases oral cancer cell proliferation in vivo and in vitro by inhibiting MMP-9 activation (21-23). However, to the best of our knowledge, the association between circulating melatonin levels and the aggressive behavior of OSCC in humans has not yet been investigated. In addition, whether melatonin may be a hormone capable of regulating MMP expression remains unknown.

The present study hypothesized that the serum melatonin level may be associated with MMP and TIMP expression in patients with OSCC. Therefore, this study aimed to determine whether serum melatonin level may be associated with MMP-9, MMP-2, TIMP-1, TIMP-2 expression levels and the clinicopathological characteristics of patients with OSCC.

\section{Materials and methods}

Patients. A total of 40 men with OSCC (mean age, $57 \pm 7$ years; age range, 46-70 years), scheduled to undergo resection surgery at the Coltea Clinical Hospital (Bucharest, Romania) between November 2014 and March 2015 were included in the present study. Samples analyses were performed at the Institute of Oncology Bucharest. The diagnosis of OSCC was based on patient history, physical examination, routine laboratory tests, endoscopy, tissue sampling and cross-sectional imaging (CT and MRI) or functional imaging with 18F-fluorodeoxyglucose positron emission tomography. The inclusion criteria were as follows: Histological diagnosis of OSCC and surgical treatment with curative intent. The exclusion criteria were as follows: i) Patients with acute or chronic infection; ii) patients with immune deficiencies; iii) patients ongoing treatments with beta-adrenergic blocking drugs (sympathetic innervation via noradrenaline has a significant role in the regulation of melatonin secretion), corticosteroids and heparin (known as MMP inhibitors) (24); iv) patients with endocrine disorders; v) patients with schizophrenia; vi) patients with burn injuries and vii) patients with previous history of chemoradiotherapy. All patients underwent primary tumor excision with adequate margins $(\geq 5 \mathrm{~mm}$ ). Radical neck dissection (functional removal of lymph nodes) was performed based on the clinical and surgical findings, which did not apply to all patients. The treatment strategies for patients were carried out according to the Coltea Clinical Hospital guidelines. Anesthesia was induced by midazolam ( $0.2 \mathrm{mg} / \mathrm{kg})$, propofol (2-2.5 mg/kg), sufentanil (0.01-0.025 mg) and sevoflurane (1-2\%). Atracurium $(0.6-1 \mathrm{mg} / \mathrm{kg}$ ) facilitated the tracheal intubation. Anesthesia was maintained with sufentanil infusion $(0.0005 \mathrm{mg} / \mathrm{kg} / \mathrm{h})$ and sevoflurane $(1-2 \%)$, whereas neuromuscular blockade was maintained with the administration of atracurium $50 \mathrm{mg}$ every $40 \mathrm{~min}$.
A total of 30 healthy men (mean age, $56 \pm 5$ years; age range, 43-69 years) with no clinical evidence of ear, nose, and throat disorders were recruited during the same period. The exclusion criteria that were applied to the patients with OSCC were also used to select the volunteers.

OSCC is more common in men compared with women, with a ratio ranging between 2:1 and 4:1 (25). Only men were included in the present study (patients and control groups) to avoid intersex variations. This study followed the principles of the Declaration of Helsinki and was approved by the Coltea Clinical Hospital Ethics Committee. All patients and volunteers signed informed consent prior to the study.

Histopathology. Clinical and histopathological data were collected from patient medical records. In the 8th edition of the American Joint Cancer Committee Cancer Staging Manual (25), an additional feature for primary tumor characterization has been introduced (26); this updated classification system has developed a modified staging system that integrates the depth of invasion (DOI) into the tumor size (T) category. Clinically, measurement of DOI requires the recognition of the basement membrane level of the closest adjacent normal mucosa and dropping of a 'plumb line' to the deepest point of tumor invasion $(25,26)$. New cut-offs have therefore been introduced according to the size and extension of the tumor ( $4 \mathrm{~cm}$ for size and $10 \mathrm{~mm}$ for depth). These boundary lines were used to classify patients into two subgroups as follows: T-DOI I $(\mathrm{n}=15)$ [small tumors with less invasive lesions $(\mathrm{T} \leq 4 \mathrm{~cm}$; $\mathrm{DOI}<10 \mathrm{~mm})$ ] and T-DOI II $(\mathrm{n}=25)$ [large tumors with invasive depth $(\mathrm{T}>4 \mathrm{~cm}$; DOI $>10 \mathrm{~mm})]$. The number of patients with positive lymph node metastases was 23 [N1 $(n=8), N 2(n=14)$, N3 $(n=1)]$, whereas 17 patients were diagnosed with lymph node-negative disease (N0). N1, N2 and $\mathrm{N} 3$ patients were analyzed as ' $\mathrm{N} 1+\mathrm{N} 2+\mathrm{N} 3$ ' or 'Yes' subgroup (lymph-node positive subgroup), whereas $\mathrm{N} 0$ remained as a single ' $\mathrm{N} 0$ ' or 'No' (lymph-node negative) subgroup. Tumors were graded as well (G1), moderately (G2) and poorly (G3) differentiated accordingly to the World Health Organization Classification of Head and Neck Tumors (27). According to the tumor grade, patients were classified as follows: $\mathrm{G} 1(\mathrm{n}=22), \mathrm{G} 2(\mathrm{n}=8)$ and $\mathrm{G} 3(\mathrm{n}=10)$.

Blood sample collection and processing. Blood specimens were collected by venipuncture into Vacutainer serum separation tubes (6 $\mathrm{ml}$; Becton, Dickinson and Company) before surgery and 2 days following surgery. Serum samples were obtained by blood clotting for $30 \mathrm{~min}$ at room temperature and centrifugation at $1,000 \times \mathrm{g}$ for $15 \mathrm{~min}$ at $4^{\circ} \mathrm{C}$. Lipemic, icteric or hemolytic specimens were excluded from this study. Following exclusion, 40 serum samples from patients with OSCC and 30 samples from healthy controls remained in the study. Serum samples were immediately aliquoted into labeled cryo-vials and stored at $-70^{\circ} \mathrm{C}$ for further analyses.

Melatonin pre-purification by solid-phase extraction. Due to the low concentration of melatonin and the coexistence of numerous other endogenous compounds in blood, such as $\mathrm{N}$-acetyl serotonin and 5-methoxi tryptamine, the determination of melatonin was an analytical challenge. Melatonin was therefore extracted from serum samples using $\mathrm{C} 18$ columns 
(R-Biopharm AG) with a recovery ranging between 87.5 and $94.8 \%$ for $10-200 \mathrm{pg}$ melatonin/ml. The solid-phase extraction procedure consisted of the following steps: i) Column conditioning with $1 \mathrm{ml}$ of bidistilled water, followed by $1 \mathrm{ml}$ of pure methanol; ii) sample application where a $0.5 \mathrm{ml}$ serum sample was passed through a C18 column that was washed with $0.5 \mathrm{ml}$ of bidistilled water and $2 \mathrm{ml}$ of water-methanol $(90: 10, \mathrm{v} / \mathrm{v})$; iii) elution of the extract where melatonin was eluted from the column with pure methanol and iv) evaporation and reconstitution of the extract where the eluate was evaporated to dryness and resuspended in $0.15 \mathrm{ml}$ of bidistilled water for further analysis. Melatonin pre-purification from serum samples was essential for high sensitivity of the analysis method (28). The procedure described above allowed melatonin detection with high sensitivity and without interference from other components in the serum.

Detection of serum melatonin, MMP-9, MMP-2, TIMP-1 and TIMP-2 by ELISA. Serum concentrations of melatonin, MMP-9, MMP-2, TIMP-1 and TIMP-2 were measured using commercially available quantitative ELISA kits from R\&D Systems, Inc. for human MMP-9 (cat. no. DMP900), MMP-2 (cat. no. DMP2F0), TIMP-1 (cat. no. DTM100) and TIMP-2 (cat. no. DTM200) and from DRG International Inc. for melatonin (cat. no. EIA-1431), according to the manufacturer's instructions.

The precision (intra-assay variation) of the assay was tested by 20 measurements of three different samples of known concentrations. The reproducibility (inter-assay variation) for the same three samples was also tested. The values of the inter-assay imprecision study were higher compared with those from the intra-assay study with coefficients of variations ranging from 3.8-16.4 and 1.9-10.3\%, respectively. The lower limits of detection were $1.6 \mathrm{pg} / \mathrm{ml}$ for melatonin, $0.156 \mathrm{ng} / \mathrm{ml}$ for MMP-9, $0.047 \mathrm{ng} / \mathrm{ml}$ for MMP-2, $0.08 \mathrm{ng} / \mathrm{ml}$ for TIMP-1 and $0.011 \mathrm{ng} / \mathrm{ml}$ for TIMP-2.

Contamination from saliva can cause falsely elevated concentrations. Since melatonin, MMP-9, TIMP-1 and TIMP-2 are present in saliva $(6,15)$, protective measures in the form of masks were taken whilst assessing the samples to prevent contamination of kit reagents while running the assay.

The serum levels of MMP-9, MMP-2, TIMP-1 and TIMP-2 were measured, and the MMP-9/TIMP-1 and MMP-2/TIMP-2 ratios were calculated. Each sample was assessed in duplicate as recommended by manufacturers in order to minimize the effects of repeated freeze-thaw cycles.

Statistical analysis. Patient data processing was performed using Microsoft Office Excel 2007 SP2, including Data Analysis Tools (Microsoft Corporation). Statistical analysis was performed using Statistica software (version 8.0; StatSoft, Inc.). Continuous variables were expressed as the means \pm standard deviation or median value with interquartile range when appropriate. The distribution of all variables was verified with the Kolmogorov-Smirnov test. The non-parametric Kruskal-Wallis test was used to compare distribution of continuous variables between different categories for independent samples (groups: OSCC vs. healthy controls; subgroups: T-DOI I vs. T-DOI II, N0 vs. N1 + N2 + N3 or G1 vs. G2 vs. G3), whereas the Wilcoxon test was used for paired samples
(OSCC group before and 2 days after surgery). Pairwise comparisons with Bonferroni post hoc corrections were used with the Kruskal-Wallis test. The correlation between circulating biomarkers was assessed using Pearson's correlation coefficient, and one-way ANOVA was used for association analysis between biomarkers and histopathological characteristics. $\mathrm{P}<0.05$ was considered to indicate a statistically significant difference.

\section{Results}

Melatonin concentration is lower in patients with OSCC compared with healthy controls. The patient distribution according to the primary tumor site was as follows: i) Tongue and base of tongue, $57.5 \%$ (23 out of $40 ; 15$ with mucosal SCC and 8 with salivary SCC); ii) gingiva, $15 \%$ (6 out of 40 ; all with salivary SCC); iii) buccal mucosa (including buccal sulcus/mucobuccal fold), $10 \%$ (4 out of $40 ; 3$ with mucosal SCC and 1 with salivary SCC); iv) soft palate and tonsil, $12.5 \%$ (5 out of 40; 3 with mucosal SCC and 2 with salivary SCC) and v) lip, $5 \%$ (2 out of 40, all with mucosal SCC) (Table I). Data from the analysis of the serum obtained from patients with OSCC (before and 2 days after surgery) and healthy controls are summarized in Table II. No significant difference was observed in patient age between OSCC and healthy controls. The circulating level of melatonin was significantly lower in patients with OSCC, both before and 2 days after surgery, compared with healthy controls $(\mathrm{P}<0.001)$. In addition, serum melatonin concentration decreased by $34.6 \%$ (from 18.2-11.9 pg/ml) 2 days after surgery, although this decrease was not statistically significant. Altered levels of the circulating ECM degradation biomarkers MMP-9, TIMP-1 and TIMP-2 $(\mathrm{P}<0.001)$ were detected in patients with OSCC compared with the controls (Table II). The serum levels of MMP-9, TIMP-1, and MMP-9/TIMP-1 and MMP-2/TIMP-2 ratios were higher, while those of TIMP-2 were lower both before and 2 days after surgery, compared with controls. No differences were observed in MMP-2 serum level between patients with OSCC and healthy controls. Furthermore, serum concentrations of MMP-9, MMP-2, and MMP-9/TIMP-1 and MMP-2/TIMP-2 ratios decreased 2 days post-surgery compared to the pre-surgical concentrations, while the levels of TIMP-1 and TIMP-2 increased (Table II).

Serum melatonin level is negatively correlated with MMP-9 and MMP-9/TIMP-1 ratio in patients with OSCC. Scatter plots presented in Fig. 1 indicated a negative correlation between serum melatonin and MMP-9 levels ( $r=-0.6371$, $\mathrm{P}<0.001$; Fig. 1A) and between serum melatonin level and the MMP-9/TIMP-1 ratio ( $r=-0.47, \mathrm{P}=0.002$; Fig. 1B). No correlations between serum melatonin and TIMP-1, MMP-2 and TIMP-2 levels or the MMP-2/TIMP-2 ratio were identified (Table III).

Low serum levels of melatonin and high serum levels of MMP-9 are associated with large tumors with invasive depth. Serum melatonin and MMP-9 levels were associated with T-DOI ( $r=-0.35, \mathrm{P}=0.027$ and $\mathrm{r}=0.33, \mathrm{P}=0.032$, respectively) in patients with OSCC (Table III). Furthermore, as presented in Table IV, serum levels of melatonin and MMP-9 were 
Table I. Patient characteristics.

\begin{tabular}{lcc}
\hline & Patient, $\mathrm{n}$ & \\
\cline { 2 - 2 } Primary tumor site & $($ Total $=40)$ & $\%$ \\
\hline Tongue and base of tongue & & \\
$\quad$ Mucosal SCC & 15 & 37.5 \\
$\quad$ Salivary SCC & 8 & 20.0 \\
$\quad$ Gingiva & & \\
$\quad$ Salivary SCC & 6 & 15.0 \\
Buccal mucosa & & \\
Mucosal SCC & 3 & 7.5 \\
Salivary SCC & 1 & 2.5 \\
Soft palate and tonsil & & \\
$\quad$ Mucosal SCC & 3 & 7.5 \\
Salivary SCC & 2 & 5.0 \\
Lip & & \\
Mucosal SCC & 2 & 5.0 \\
\hline
\end{tabular}

SCC, squamous cell carcinoma.

significantly lower and higher, respectively, in the T-DOI II subgroup compared with the T-DOI I subgroup ( $\mathrm{P}=0.012$ and $\mathrm{P}=0.033$, respectively).

The correlation between serum melatonin and ECM biomarker levels was also investigated in the two subgroups of patients with OSCC. The results presented in Fig. 2 demonstrated a negative correlation between serum melatonin and MMP-9 levels ( $\mathrm{r}=-0.7539, \mathrm{P}=0.001$ in T-DOI $\mathrm{I}$ and $\mathrm{r}=-0.4407$, $\mathrm{P}=0.027$ in T-DOI II). Furthermore, melatonin was negatively correlated with MMP-9/TIMP-1 ratio in the T-DOI II subgroup $(\mathrm{r}=-0.4818, \mathrm{P}=0.015$; Fig. 2C).

Low serum levels of melatonin and high serum levels of MMP-9 are associated with lymph node metastasis. Melatonin and MMP-9 serum levels were associated with lymph node involvement $(\mathrm{r}=-0.56, \mathrm{P}<0.001$ and $\mathrm{r}=0.34, \mathrm{P}=0.031$, respectively) in patients with OSCC (Table III). Serum levels of melatonin and MMP-9 were significantly lower and higher, respectively, in the lymph node-positive subgroup compared with the lymph node-negative subgroup $(\mathrm{P}<0.001$ and $\mathrm{P}=0.029$, respectively; Table IV). Serum TIMP-1, MMP-2 and TIMP-2 levels and the MMP-2/TIMP-2 ratio were not significantly different in patients with negative lymph node metastases compared with patients with positive lymph node metastases. Regarding the analysis of patients with OSCC according to tumor size or the extent of the tumor, melatonin was negatively correlated with serum MMP-9 levels in both subgroups $(r=-0.5260$, $\mathrm{P}=0.03$ in lymph node-negative subgroup; Fig. $3 \mathrm{~A} ; \mathrm{r}=-0.6259$, $\mathrm{P}=0.01$ in lymph node-positive subgroup; Fig. $3 \mathrm{~B}$ ) and with the MMP-9/TIMP-1 ratio only in the subgroup of patients with positive lymph node metastases $(r=-0.5284, \mathrm{P}=0.02$ in lymph node-positive subgroup; Fig. 3C).

Association between melatonin, ECM biomarkers and tumor grading. No significant differences were observed between the serum levels of all biomarkers measured in G1, G2 or G3 subgroups of patients with OSCC, with the exception of MMP-2 ( $\mathrm{P}=0.028$; Table IV). Regarding correlations between melatonin and ECM degradation biomarkers, strong negative correlations were identified between serum melatonin and MMP-9 levels in the $\mathrm{G} 1$ and $\mathrm{G} 2$ subgroups $(\mathrm{r}=-0.65, \mathrm{P}=0.001$ and $\mathrm{r}=-0.79, \mathrm{P}=0.018$, respectively) and between serum melatonin levels and the MMP-9/TIMP-1 ratio only in the G1 subgroup ( $\mathrm{r}=-0.47, \mathrm{P}=0.025)$ (Table $\mathrm{V}$ ).

No correlations were identified between serum melatonin and TIMP-1, MMP-2 and TIMP-2 levels or the MMP-2/TIMP-2 ratio in all subgroups characterized according to tumor size and DOI, lymph node involvement or tumor grading (data not shown).

\section{Discussion}

The present study demonstrated that serum melatonin levels were significantly lower in patients with OSCC before and 2 days after surgery compared with healthy controls. In addition, serum melatonin level was negatively correlated with serum MMP-9 level and the MMP-9/TIMP-1 ratio in patients with OSCC. Low serum levels of melatonin and high serum levels of MMP-9 were associated with invasion depth and lymph node metastasis.

Previous studies have reported that the disruption of blood melatonin level is associated with an increased risk of various types of cancer occurrence, including breast, prostate, ovarian and colon cancer (16-19). The results of the present study demonstrated that serum melatonin level was significantly lower in patients with OSCC compared with healthy controls, which was consistent with results from previous studies reporting that low serum melatonin level was associated with an increased risk of breast (16), prostate (17), ovarian (18) or colon (19) cancer. In addition, median circulating melatonin concentration in the T DOI I $(\leq 36.8 \mathrm{pg} / \mathrm{ml})$ or negative lymph node metastasis $(\leq 37.9 \mathrm{pg} / \mathrm{ml})$ subgroups of patients with OSCC were similar to those obtained by Yang et al (16) in patients with breast cancer $(\leq 39.5 \mathrm{pg} / \mathrm{ml})$.

Previous studies have reported a modest reduction in melatonin secretion on the first day following different types of surgery, such as orthopedic and abdominal, compared with pre-surgery levels, as well as a high increase in melatonin secretion on the second day following surgery $(29,30)$. The decrease in melatonin and MMP-9 serum level one day post-surgery may be due to the effects of general anesthesia, particularly the administration of propofol $(31,32)$. To eliminate the influence of propofol on the circadian rhythm of melatonin and MMP-9 in patients with OSCC in the present study, blood was collected on the second day post-surgery. In contrast to previous studies $(29,30)$, the present study demonstrated that serum melatonin level decreased by $34.6 \% 2$ days following surgery, although this decrease was not statistically significant. These differences may be due to the various sites of surgery [oral cavity in the present study vs. knee (29) and abdomen (30) in previous studies]. OSCC affects the preferential sites of melatonin reactivity in the oral cavity that are the salivary glands, including parotid, submandibular and sublingual glands. In the present study, $42.5 \%$ of patients with OSCC (17 out of 40) were diagnosed with salivary SCC. 
Table II. Biochemical data in patients with OSCC and healthy controls.

\begin{tabular}{|c|c|c|c|c|c|c|}
\hline \multirow[b]{2}{*}{ Variables } & \multicolumn{2}{|c|}{ OSCC } & \multirow[b]{2}{*}{ Control } & \multirow[b]{2}{*}{ P-value } & \multirow[b]{2}{*}{ P-value ${ }^{d}$} & \multirow[b]{2}{*}{ P-value } \\
\hline & Pre-surgery & Post-surgery & & & & \\
\hline Number, $\mathrm{n}$ & 40 & 40 & 30 & $>0.999$ & 0.452 & 0.452 \\
\hline Age, years $^{\mathrm{a}}$ & $57 \pm 7$ & $57 \pm 7$ & $56 \pm 5$ & $>0.999$ & 0.670 & 0.670 \\
\hline Melatonin, $\mathrm{pg} / \mathrm{ml}^{\mathrm{b}}$ & $18.2(11.0-39.2)$ & $11.9(8.7-31.4)$ & $47.6(37.7-66.4)$ & 0.230 & $<0.001^{\mathrm{f}}$ & $<0.001^{\mathrm{f}}$ \\
\hline MMP-9, ng/ml ${ }^{\mathrm{b}}$ & $1,619(914-1,964)$ & $1,402(877-2,001)$ & $291(211-330)$ & 0.939 & $<0.001^{\mathrm{f}}$ & $<0.001^{\mathrm{f}}$ \\
\hline TIMP-1, ng/ml & $262(213-430)$ & $351(255-452)$ & $164(121-201)$ & 0.132 & $<0.001^{\mathrm{f}}$ & $<0.001^{\mathrm{f}}$ \\
\hline MMP-9/TIMP-1 ${ }^{\mathrm{b}}$ & $4.8(3.1-7.4)$ & $3.9(2.6-6.2)$ & $1.7(1.5-1.8)$ & 0.129 & $<0.001^{\mathrm{f}}$ & $<0.001^{\mathrm{f}}$ \\
\hline MMP-2, ng/ml & $160(130-197)$ & $149(127-196)$ & $150(122-180)$ & 0.632 & 0.658 & 0.991 \\
\hline TIMP-2, ng/ml ${ }^{\mathrm{b}}$ & $57.5(51.6-70.5)$ & $62.5(49.9-76)$ & $89.5(79.7-101)$ & 0.289 & $<0.001^{\mathrm{f}}$ & $<0.001^{\mathrm{f}}$ \\
\hline MMP-2/TIMP-2 ${ }^{\mathrm{b}}$ & $2.8(2.4-3.2)$ & $2.6(2.2-2.9)$ & $1.6(1.3-1.9)$ & 0.392 & $<0.001^{\mathrm{f}}$ & $<0.001^{\mathrm{f}}$ \\
\hline
\end{tabular}

Table III. Association between circulating biomarkers and clinicopathological characteristics of patients with OSCC.

\begin{tabular}{lcccccccccc}
\hline Variables & Melatonin & P-value & MMP-9 & P-value & MMP-2 & P-value & TIMP-1 & P-value & TIMP-2 & P-value \\
\hline Melatonin & - & - & -0.6371 & $<0.001$ & -0.1766 & 0.276 & 0.0238 & 0.884 & -0.2947 & 0.065 \\
MMP-9 & -0.6371 & $<0.001$ & - & - & 0.0006 & 0.997 & 0.2780 & 0.082 & 0.2093 & 0.195 \\
TIMP-1 & 0.0238 & 0.884 & 0.2780 & 0.082 & 0.1853 & 0.252 & - & - & 0.2455 & 0.127 \\
MMP-9/TIMP-1 & -0.4700 & 0.002 & 0.4813 & 0.002 & -0.1735 & 0.284 & -0.5345 & $<0.001$ & -0.0035 & 0.983 \\
MMP-2 & -0.1766 & 0.276 & 0.0009 & 0.997 & - & - & 0.1853 & 0.252 & 0.6796 & $<0.001$ \\
TIMP-2 & -0.2947 & 0.065 & 0.2093 & 0.195 & 0.6796 & $<0.001$ & 0.2455 & 0.127 & - \\
MMP-2/TIMP-2 & 0.1760 & 0.277 & -0.2307 & 0.152 & 0.3380 & 0.033 & -.0 .0822 & 0.614 & -0.4350 & 0.005 \\
T-DOI & -0.3494 & 0.027 & 0.3321 & 0.032 & 0.2494 & 0.121 & 0.1669 & 0.303 & 0.0852 & 0.601 \\
Nodal involvement & -0.5583 & $<0.001$ & 0.3422 & 0.031 & 0.1760 & 0.277 & -.0160 & 0.922 & 0.1180 & 0.468 \\
Differentiation & -0.1309 & 0.421 & 0.1646 & 0.310 & 0.4072 & 0.009 & 0.2257 & 0.161 & 0.1448 & 0.373
\end{tabular}

T, tumor size; DOI, depth of invasion; MMP-9, matrix metalloproteinase-9; MMP-2, matrix metalloproteinase-2; TIMP-1, tissue inhibitor of metalloproteinase-1; TIMP-2, tissue inhibitor of metalloproteinase-2.
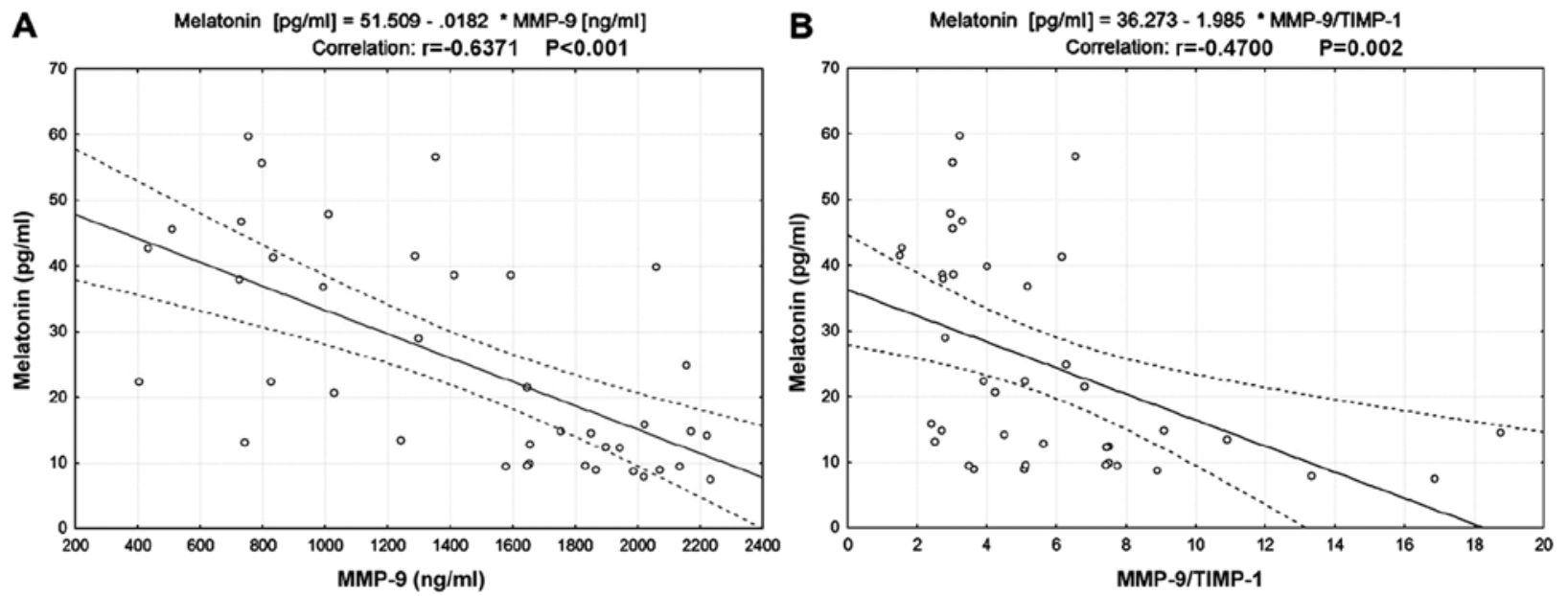

Figure 1. Correlation between melatonin, MMP-9 and TIMP-1 in patients with OSCC.(A) Correlation between serum melatonin and MMP-9 levels. (B) Correlation between serum melatonin level and the MMP-9/TIMP-1 ratio. MMP-9, matrix metalloproteinase 9; TIMP-1, tissue inhibitor of metalloproteinase 1. 
Regarding MMP-9, the results of the present study were consistent with previous studies $(7,9)$ that reported a reduction in serum MMP-9 levels in patients with OSCC following surgery. The present study demonstrated a decrease in serum MMP-9 levels 2 days post-surgery, suggesting that MMP-9 may be considered as a marker of tumor presence, which has been previously demonstrated $(7,9,33)$; however, the difference between serum MMP-9 level before and after surgery was not significant. In addition, previous studies have demonstrated that MMP-9 serum levels may reflect its expression in resected tumor tissue $(7,9,33)$. The lack of statistical significance in the present study may be due to the fact that the 2-day post-surgical interval may be too short to observe a total recovery of a normal serum MMP-9 concentration. A significant decrease in the serum MMP-9 level becomes evident only 1 month following surgery (33).

The results from the present study suggested that radical surgery with excision of the primary tumor, including salivary glands, reduced not only serum MMP-9 levels, but also serum melatonin level. High MMP-9 and melatonin expression levels have been observed in excised tumor tissues $(7,9,15)$. These findings suggest that melatonin suppression may be prevented by treatment with melatonin supplements, and that a prospective clinical trial assessing the therapeutic potential of melatonin in patients with OSCC may be useful.

The present study hypothesized that melatonin may be able to regulate MMP expression and activity. To the best of our knowledge, the present study was the first to demonstrate the association between melatonin, MMPs, TIMPs and the clinicopathological characteristics of patients with OSCC.

Our group previously reported that the imbalance between MMP-9 and TIMP-1 serum levels may serve a crucial role in the metastatic spread of head and neck cancer cells via lymphatic pathways $(6,8)$. Furthermore, MMP-2 and TIMP-2 may be used as markers of tumor differentiation in head and neck squamous cell carcinoma $(2,6)$. In the present study, serum MMP-9 level and the MMP-9/TIMP-1 ratio were significantly higher, whereas serum melatonin level was significantly lower in patients with OSCC compared with healthy controls. These results were consistent with those from Lin et al (34), who demonstrated that melatonin receptor type 1A (MTNR1A) was reduced in 618 patients with oral cancer compared with 560 non-cancer controls, as well as those from other studies reporting that an increase in serum MMP-9 level or the MMP-9/TIMP-1 ratio may enhance invasion in head and neck cancers $(7,9)$. Inhibition of MMP-9 activity may therefore reduce cancer metastasis in patients with OSCC $(34,35)$.

In the present study, serum melatonin level was negatively correlated with serum MMP-9 level and the MMP-9/TIMP-1 ratio. Similarly, previous studies reported a negative correlation between melatonin, MTNR1A and MMP-9 expression in healthy kidney and renal cell carcinoma tissues (36), and a $50 \%$ inhibitory effect of melatonin on the induction and catalytic activity of MMP-9 in the human gastric adenocarcinoma cell line AGS (29). Lin et al (36) have demonstrated that melatonin can inhibit NF- $\kappa \mathrm{B}$-mediated MMP-9 transcription and cancer cell invasion by targeting the Akt-Erk/JNK pathways in renal cell carcinoma. Furthermore, in a human gastric adenocarcinoma cell line, melatonin inhibits MMP-9 
Table V. Correlation between circulating biomarkers in G1, G2 and G3 subgroups of patients with OSCC.

\begin{tabular}{|c|c|c|c|c|c|c|c|c|c|}
\hline \multirow{2}{*}{$\frac{\text { Subgroups }}{\text { Variables }}$} & \multicolumn{3}{|c|}{ G1 } & \multicolumn{3}{|c|}{ G2 } & \multicolumn{3}{|c|}{ G3 } \\
\hline & Melatonin & MMP-9 & $\begin{array}{c}\text { MMP-9/ } \\
\text { TIMP-1 }\end{array}$ & Melatonin & MMP-9 & $\begin{array}{l}\text { MMP-9/ } \\
\text { TIMP-1 }\end{array}$ & Melatonin & MMP-9 & $\begin{array}{l}\text { MMP-9/ } \\
\text { TIMP-1 }\end{array}$ \\
\hline Melatonin & - & $\begin{array}{l}-0.6576 \\
P=0.001\end{array}$ & $\begin{array}{l}-0.4755 \\
\mathrm{P}=0.025\end{array}$ & - & $\begin{array}{c}-0.7948 \\
P=0.018\end{array}$ & $\begin{array}{l}-0.6149 \\
\mathrm{P}=0.105\end{array}$ & - & $\begin{array}{c}-0.5379 \\
\mathrm{P}=0.109\end{array}$ & $\begin{array}{l}-0.6185 \\
P=0.057\end{array}$ \\
\hline MMP-9 & $\begin{array}{l}-0.6576 \\
P=0.001\end{array}$ & - & $\begin{array}{c}0.5641 \\
\mathrm{P}=0.006\end{array}$ & $\begin{array}{l}-0.7948 \\
\mathrm{P}=0.018\end{array}$ & - & $\begin{array}{c}0.6386 \\
\mathrm{P}=0.038\end{array}$ & $\begin{array}{l}-0.5379 \\
P=0.109\end{array}$ & - & $\begin{array}{l}0.06051 \\
\mathrm{P}=0.024\end{array}$ \\
\hline $\begin{array}{l}\text { MMP-9/ } \\
\text { TIMP-1 }\end{array}$ & $\begin{array}{l}-0.4755 \\
\mathrm{P}=0.025\end{array}$ & $\begin{array}{c}0.5641 \\
\mathrm{P}=0.006\end{array}$ & - & $\begin{array}{l}-0.6149 \\
P=0.105\end{array}$ & $\begin{array}{c}0.6386 \\
\mathrm{P}=0.038\end{array}$ & - & $\begin{array}{l}-0.6185 \\
P=0.057\end{array}$ & $\begin{array}{l}0.06051 \\
P=0.024\end{array}$ & - \\
\hline
\end{tabular}

MMP-9, matrix metalloproteinase 9; TIMP-1, tissue inhibitor of metalloproteinase-1; G1 subgroup-well differentiated (low grade) tumors; G2 subgroup-moderately differentiated (intermediate grade) tumors; G3 subgroup-poorly differentiated (high grade) tumors.
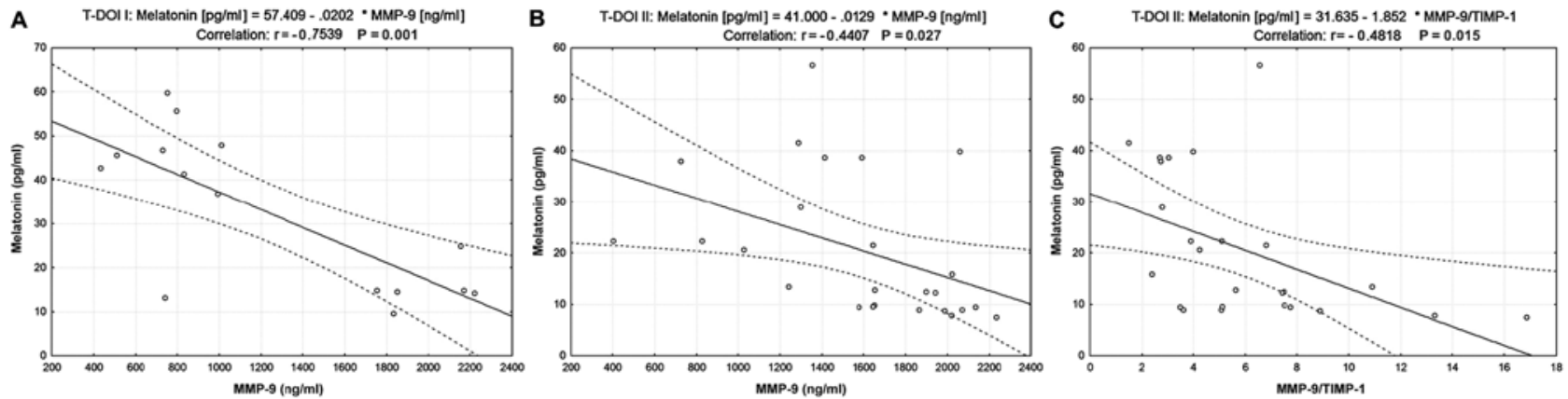

Figure 2. Correlation between melatonin, MMP-9 and TIMP-1 in two subgroups of patients with OSCC classified according to T and DOI. (A) Correlation between serum melatonin and MMP-9 y levels in the T-DOI I subgroup. (B) Correlation between serum melatonin and MMP-9 levels in the T-DOI II subgroup. (C) Correlation between serum melatonin level and the MMP-9/TIMP-1 ratio in the T-DOI II subgroup. DOI, depth of invasion; MMP-9, matrix metalloproteinase 9; T, tumor size; TIMP-1, tissue inhibitor of metalloproteinase 1.
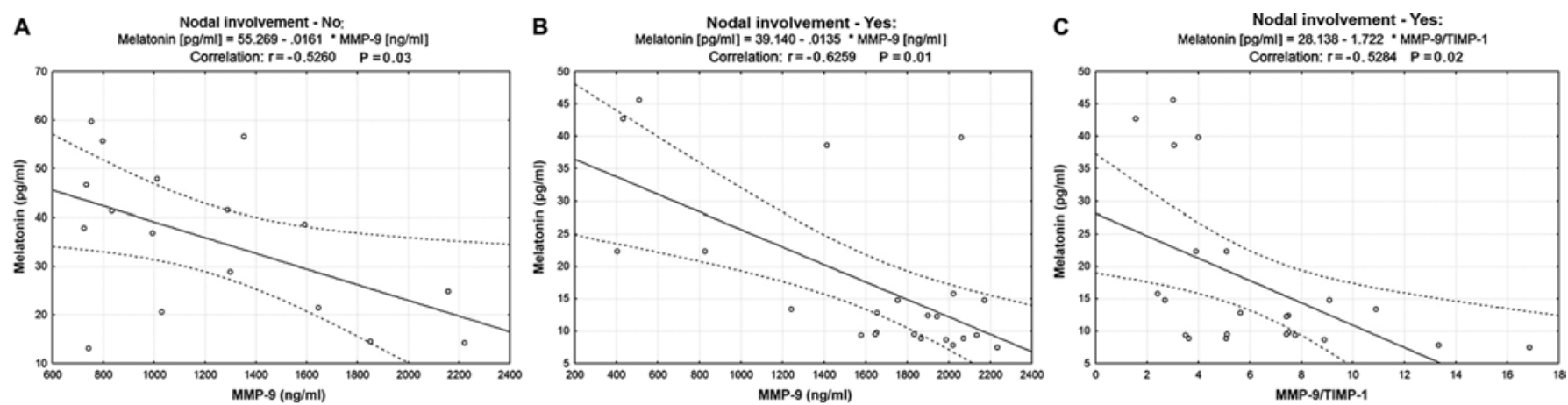

Figure 3. Correlation between melatonin, MMP-9 and TIMP-1 in two subgroups of patients with OSCC classified according to lymph node involvement. (A) Correlation between serum melatonin and MMP-9 levels in the lymph node-negative subgroup. (B) Correlation between serum melatonin and MMP-9 levels in the lymph node-positive subgroup. (C) Correlation between serum melatonin level and the MMP-9/TIMP-1 ratio in the lymph node-positive subgroup. MMP-9, matrix metalloproteinase 9; TIMP-1, tissue inhibitor of metalloproteinase 1.

activity by binding to its active site and by interacting with key residues within its catalytic site, including three histidines that form the coordination complex with zinc, proline 421 and alanine 191 (37).

Numerous in vitro studies have reported that MMP-9 is a critical target of melatonin in the regulation of oral cancer metastasis (20-23). A number of mechanisms by which melatonin could repress MMP-9 expression have been revealed.
For example, melatonin can inhibit the motility of oral cancer cells (HSC-3 and OECM-1), attenuating MMP-9 expression and activity by reducing histone acetylation on the promoter of MMP-9 gene (21). Furthermore, melatonin can suppress nasopharyngeal carcinoma cell migration by regulating MMP-9 gene expression and inhibiting the DNA-binding ability of the transcription factor specificity protein-1 (22). Lu et al (20) have demonstrated that melatonin treatment 
of the OSCC cell lines SCC-9 and SCC-25 can reduce the survival and migration of OSCC-associated neutrophils and suppress the tumor-associated neutrophil release of C-X-C motif chemokine ligand $8, \mathrm{C}-\mathrm{C}$ motif chemokine ligand 2 , C-C motif chemokine ligand 4 and MMP-9 by blocking p38 MAPK and Akt signaling.

To the best of our knowledge, no clinical studies exploring the effect of melatonin and MMPs mediated ECM remodeling on the susceptibility of developing OSCC and histological features of OSCC are presently available. Lu et al (20) have reported that intensive infiltration of tumor-associated neutrophils in OSCC tissue specimens is associated with advanced stage, lymphatic metastasis and poor prognosis of patients with OSCC. Furthermore, the in vitro study by Lu et al (20) reported that the pro-motility and pro-angiogenesis effects of OSCC-associated neutrophils decreased by melatonin were dependent of MMP-9 suppression in OSCC. In the present study, the results of the assessment of melatonin and MMP-9 serum levels in patients with OSCC demonstrated that low levels of melatonin and high levels of MMP-9 were associated with advanced tumor stage and lymphatic metastasis.

Tumor size and the extent of the primary tumor are a well-known prognostic factor for numerous types of cancer, including OSCC, and larger tumors predict a worse prognosis $(25,38)$. The results from a recent study suggest that patient prognosis worsens when DOI increases, and that DOI is a better predictive parameter compared with the $\mathrm{T}$ category (26). The results of the present study demonstrated that low levels of melatonin and high levels of MMP-9 were associated with large tumors with high invasive depth (T $>4 \mathrm{~cm}$; DOI $>10 \mathrm{~mm}$ ). In addition, serum melatonin and MMP-9 levels were significantly lower and higher in the T-DOI II subgroup compared with the T-DOI I, respectively. In both T-DOI subgroups, serum melatonin level was negatively correlated with serum MMP-9 level. Furthermore, melatonin was inversely associated with T-DOI in patients with OSCC. In line with the findings of the present study, Nakamura et al (39) analyzed 50 primary OSCC tumors by immunohistochemistry and reported that the absence of immunoreactive MTNR1A is negatively associated with the T category of the Tumor-Node-Metastasis (TNM) classification.

Regarding metastatic dissemination to regional lymph nodes, the present study demonstrated that melatonin was inversely associated with the presence of lymph node metastases in patients with OSCC. By contrast, Nakamura et al (39) reported that the expression of MTNR1A was not associated with the $\mathrm{N}$ category of the TNM classification, suggesting that the reduced MTNR1A expression and MTNR1A inactivation may occur in a specific subgroup of OSCC, even at an earlier stage, and contribute to the higher proliferative and/or survival activity of tumor cells in this subgroup. The findings of the study by Nakamura et al (39) may also be valid in the present study, although this study demonstrated that melatonin was associated with the $\mathrm{N}$ category, since the loss of melatonin occurred in an earlier stage in the lymph-node negative subgroup, and the serum concentration was lower compared with that in controls. In addition, serum melatonin level was negatively correlated with serum MMP-9 level even in the lymph node-negative subgroup. The present study also demonstrated that serum melatonin level in the lymph node-positive subset of patients was significantly lower compared with patients in the lymph node-negative subgroup $(\mathrm{P}<0.001)$. These results suggested that a reduced serum level of melatonin may be associated with the metastatic rate in cervical lymph nodes in patients with OSCC.

An essential feature of the invasive phenotype of a tumor is its ability to produce MMPs $(4,6,7)$. In the present study, MMP-2 and MMP-9 serum levels were higher in poorly differentiated tumors, corresponding to an advanced stage of neoplasia, compared with well-differentiated tumors, which are normally associated with better prognosis. Furthermore, serum melatonin level was not associated with the degree of differentiation of the tumor cells. However, serum melatonin and MMP-9 levels were negatively correlated in the low- and intermediate-grade tumor subgroup, but not in the high-grade tumor subgroup.

The present study presented certain limitations. Firstly, this study was a single-center, retrospective cohort analysis, with a small number of patients included. However, despite the small sample size, the population was homogeneous and included only men in both groups. The samples were collected during the same season (winter) and at the same time in the morning in order to avoid any artefacts. Secondly, immunohistochemistry to detect melatonin and ECM biomarkers in OSCC tissue specimens could not be performed, and their association was therefore not established. Further studies investigating the immunohistochemical expression of melatonin and ECM biomarkers in OSCC tissue specimens are required. Thirdly, cell experiments to validate the association between melatonin and MMP-9 were not conducted. This association should be investigated in future studies.

In summary, the results of the present study demonstrated that serum melatonin level was negatively correlated with serum MMP-9 level, tumor size, depth of invasion and metastasis in patients with OSCC. The results of this retrospective study indicated that melatonin may be considered as a predictive biomarker of cancer cell proliferation and metastasis and a potential therapeutic agent for patients with OSCC. Further investigation is required to confirm these findings.

\section{Acknowledgements}

Not applicable.

\section{Funding}

No funding was received.

\section{Availability of data and materials}

The datasets used and/or analyzed during the current study are available from the corresponding author on reasonable request.

\section{Authors' contributions}

AES and AZCA conceived the study and planned the experiments. Experiments were performed by AES. AES and MMS 
performed statistical analysis. AES, AZCA, MMS, APS, CN and DCG contributed to the interpretation of the results. AS wrote the manuscript. All authors provided critical feedback and contributed to the design of this study, data analysis and manuscript writing.

\section{Ethics approval and consent to participate}

The present study conformed to the principles of The Declaration of Helsinki and was approved by the Coltea Clinical Hospital Ethics Committee (Bucharest, Romania). Written informed consent was obtained from all patients and volunteers prior to the study start.

\section{Patient consent for publication}

Not applicable.

\section{Competing interests}

The authors declare that they have no competing interests.

\section{References}

1. Yeh CM, Su SC, Lin CW, Yang WE, Chien MH, Reiter RJ and Yang SF: Melatonin as a potential inhibitory agent in head and neck cancer. Oncotarget 8: 90545-90556, 2017.

2. Stanciu AE, Zamfir-Chiru-Anton A, Stanciu MM, Popescu CR, Gheorghe DC and Nitipir C: Serum matrix metalloproteinase-2 in head and neck squamous cell carcinoma is associated with tumor differentiation. Rom Biotech Lett 22: 12419-12426, 2017.

3. Koziorowski J, Stanciu AE, Gomez-Vallejo V and Llop J: Radiolabeled nanoparticles for cancer diagnosis and therapy. Anticancer Agents Med Chem 17: 333-354, 2017.

4. Gialeli C, Theocharis AD and Karamanos NK: Roles of matrix metalloproteinases in cancer progression and their pharmacological targeting. FEBS J 278: 16-27, 2011.

5. Stanciu AE, Vatasescu RG, Stanciu MM, Serdarevic N and Dorobantu M: The role of pro-fibrotic biomarkers in paroxysmal and persistent atrial fibrillation. Cytokine 103: 63-68, 2018.

6. Stanciu AE, Zamfir-Chiru-Anton A, Stanciu MM, Popescu CR and Gheorghe DC: Imbalance between matrix metalloproteinases and tissue inhibitors of metalloproteinases promotes invasion and metastasis of head and neck squamous cell carcinoma. Clin Lab 63: 1613-1620, 2017.

7. Patel BP, Shah SV, Shukla SN, Shah PM and Patel PS: Clinical significance of MMP-2 and MMP-9 in patients with oral cancer Head Neck 29: 564-572, 2007.

8. Stanciu AE, Zamfir-Chiru-Anton A, Stanciu MM, Popescu CR and Gheorghe DC: Serum level of matrix metalloproteinase-9 in patients with head and neck squamous cell carcinoma. Clin Lab 62: 1569-1574, 2016.

9. Burduk PK, Sawicki P, Szylberg L, Bodnar M and Marszalek A: Expression of matrix metalloproteinase-2/9 and tissue inhibitor of metalloproteinase-1/2 as predictive factors in oropharyngeal squamous cell carcinoma. Iran J Otorhinolaryngol 31: 153-161, 2019.

10. Hanedan Uslu G, Canyilmaz E, Serdar L and Ersöz S: Protective effects of genistein and melatonin on mouse liver injury induced by whole-body ionising radiation. Mol Clin Oncol 10: 261-266, 2019.

11. Laurent V, Sengupta A, Sánchez-Bretaño A, Hicks D and Tosini G: Melatonin signaling affects the timing in the daily rhythm of phagocytic activity by the retinal pigment epithelium. Exp Eye Res 165: 90-95, 2017.

12. Siah KT, Wong RK and Ho KY: Melatonin for the treatment of irritable bowel syndrome. World J Gastroenterol 20: 2492-2498, 2014.

13. Slominski A, Fischer TW, Zmijewski MA, Wortsman J, Semak I, Zbytek B, Slominski RM and Tobin DJ: On the role of melatonin in skin physiology and pathology. Endocrine 27: $137-148,2005$
14. Conti A, Conconi S, Hertens E, Skwarlo-Sonta K, Markowska M and Maestroni JM: Evidence for melatonin synthesis in mouse and human bone marrow cells. J Pineal Res 28: 193-202, 2000.

15. Isola M and Lilliu MA: Melatonin localization in human salivary glands. J Oral Pathol Med 45: 510-515, 2016.

16. Yang WS, Deng Q, Fan WY, Wang WY and Wang X: Light exposure at night, sleep duration, melatonin, and breast cancer: A dose-response analysis of observational studies. Eur J Cancer Prev 23: 269-276, 2014.

17. Tai SY, Huang SP, Bao BY and Wu MT: Urinary melatonin-sulfate/cortisol ratio and the presence of prostate cancer: A case control study. Sci Rep 6: 29606, 2016.

18. Zhao M, Wan JY, Zeng K, Tong M, Lee AC, Ding JX and Chen Q: The reduction in circulating melatonin level may contribute to the pathogenesis of ovarian cancer: A retrospective study. J Cancer 7: 831-836, 2016.

19. Chok KC, Ng CH, Koh RY, Ng KY and Chye SM: The potential therapeutic actions of melatonin in colorectal cancer. Horm Mol Biol Clin Investig: May 29, 2019 (Epub ahead of print). doi: 10.1515/hmbci-2019-0001.

20. Lu H, Wu B, Ma G, Zheng D, Song R, Huang E, Mao M and $\mathrm{Lu}$ B: Melatonin represses oral squamous cell carcinoma metastasis by inhibiting tumor-associated neutrophils. Am J Transl Res 9: 5361-5374, 2017.

21. Yeh CM, Lin CW, Yang JS, Yang WE, Su SC and Yang SF: Melatonin inhibits TPA-induced oral cancer cell migration by suppressing matrix metalloproteinase-9 activation through the histone acetylation. Oncotarget 7: 21952-21967, 2016.

22. Ho HY, Lin CW, Chien MH, Reiter RJ, Su SC, Hsieh YH and Yang SF: Melatonin suppresses TPA-induced metastasis by downregulating matrix metalloproteinase-9 expression through JNK/SP-1 signaling in nasopharyngeal carcinoma. J Pineal Res 61: 479-492, 2016.

23. Yang CY, Lin CK, Tsao CH, Hsieh CC, Lin GJ, Ma KH, Shieh YS, Sytwu HK and Chen YW: Melatonin exerts anti-oral cancer effect via suppressing LSD1 in patient-derived tumor xenograft models. Oncotarget 8: 33756-33769, 2017.

24. Mannello F, Jung K, Tonti GA and Canestrari F: Heparin affects matrix metalloproteinases and tissue inhibitors of metalloproteinases circulating in peripheral blood. Clin Biochem 41: 1466-1473, 2008

25. Amin MB, Edge S, Greene F, Byrd DR, Brookland RK, Washington MK, Gershenwald JE. Compton CC, Hess KR, Sullivan DC, et al: AJCC Cancer Staging Manual, eighth edition (2017) published by Springer Science and Business Media LLC (springer.com). AJCC Cancer Staging Manual. 8th edition. Springer, New York, NY, 2017.

26. Lydiatt WM, Patel SG, O'Sullivan B, Brandwein MS, Ridge JA, Migliacci JC, Loomis AM and Shah JP: Head and Neck cancers-major changes in the American Joint Committee on cancer eighth edition cancer staging manual. CA Cancer J Clin 67: 122-137, 2017

27. El-Naggar AK, Chan JKC, Grandis JR, Takata T and Slootweg PJ: WHO Classification of Head and Neck Tumours. WHO Classification of Tumours. 4th edition. Volume 9. WHO Press, Geneva, 2017.

28. Kennaway DJ: A critical review of melatonin assays: Past and present. J Pineal Res 67: e12572, 2019.

29. Karkela J, Vakkuri O, Kaukinen S, Huang WQ and Pasanen M: The influence of anaesthesia and surgery on the circadian rhythm of melatonin. Acta Anaesthesiol Scand 46: 30-36, 2002.

30. Vacas S, Kurien P and Maze M: Sleep and Anesthesia Common mechanisms of action. Sleep Med Clin 8: 1-9, 2013.

31. Norouzi A, Fateh S, Modir H, Kamali A and Akrami L: Premedication effect of melatonin on propofol induction dose for anesthesia, anxiety, orientation and sedation after abdominal surgery: A double-blinded randomized trial. Med Gas Res 9: 62-67, 2019.

32. Wang G, Liu J, Gao J and Zheng X: Comparison of the effects of sevoflurane and propofol anesthesia on pulmonary function, MMP-9 and postoperative cognition in patients receiving lung cancer resection. Oncol Lett 17: 3399-3405, 2019.

33. Vasaturo F, Solai F, Malacrino C, Nardo T, Vincenzi B, Modesti M and Scarpa S: Plasma levels of matrix metalloproteinases 2 and 9 correlate with histological grade in breast cancer patients. Oncol Lett 5: 316-320, 2013. 
34. Lin FY, Lin CW, Yang SF, Lee WJ, Lin YW, Lee LM, Chang JL, Weng WC, Lin $\mathrm{CH}$ and Chien MH: Interactions between environmental factors and melatonin receptor type $1 \mathrm{~A}$ polymorphism in relation to oral cancer susceptibility and clinicopathologic development. PLoS One 10: e0121677, 2015.

35. Lin FY, Hsieh YH, Yang SF, Chen CT, Tang CH, Chou MY, Chuang YT, Lin CW and Chen MK: Resveratrol suppresses TPA-induced matrix metalloproteinase-9 expression through the inhibition of MAPK pathways in oral cancer cells. J Oral Pathol Med 44: 699-706, 2015.

36. Lin YW, Lee LM, Lee WJ, Chu CY, Tan P, Yang YC, Chen WY, Yang SF, Hsiao M and Chien MH: Melatonin inhibits MMP-9 transactivation and renal cell carcinoma metastasis by suppressing Akt-MAPKs pathway and NF- $\kappa \mathrm{B}$ DNA-binding activity. J Pineal Res 60: 277-290, 2016.
37. Rudra DS,Pal U, Maiti NC, Reiter RJ and Swarnakar S: Melatonin inhibits matrix metalloproteinase- 9 activity by binding to its active site. J Pineal Res 54: 398-405, 2013.

38. Stanciu AE, Zamfir-Chiru-Anton A, Stanciu MM, Pantea Stoian A, Jinga V, Nitipir C, Bucur A, Pituru TS, Arsene AL, Dragoi CM, et al: Clinical significance of serum melatonin in predicting the severity of oral squamous cell carcinoma. Oncol Lett 19: 1537-1543, 2019.

39. Nakamura E, Kozaki K, Tsuda H, Suzuki E, Pimkhaokham A, Yamamoto G, Irie T, Tachikawa T, Amagasa T, Inazawa J and Imoto I: Frequent silencing of a putative tumor suppressor gene melatonin receptor $1 \mathrm{~A}$ (MTNR1A) in oral squamous-cell carcinoma. Cancer Sci 99: 1390-400, 2008. 